\title{
Shock structure and shock heating in the Galactic central molecular zone
}

\author{
Jürgen Ott ${ }^{1,2}$, Michael Burton ${ }^{3}$, Paul Jones ${ }^{3}$ and David S. Meier ${ }^{2,1}$ \\ ${ }^{1}$ National Radio Astronomy Observatory, P. O. Box O, 1003 Lopezville Road, Socorro, \\ NM 87801, USA; email: jott@nrao.edu \\ ${ }^{2}$ Department of Physics, New Mexico Institute of Mining and Technology, Socorro, NM 87801, \\ USA; email: dmeier@nmt.edu \\ ${ }^{3}$ School of Physics, University of New South Wales, Sydney NSW 2052, Australia; email:
}

M. Burton@unsw.edu.au, paulcojones@gmail.com

\begin{abstract}
We present maps of a large number of dense molecular gas tracers across the central molecular zone of our Galaxy. The data were taken with the CSIRO/CASS Mopra telescope in Large Projects in the $1.3 \mathrm{~cm}, 7 \mathrm{~mm}$, and $3 \mathrm{~mm}$ wavelength regimes. Here, we focus on the brightness of the shock tracers $\mathrm{SiO}$ and $\mathrm{HNCO}$, molecules that are liberated from dust grains under strong $(\mathrm{SiO})$ and weak (HNCO) shocks. The shocks may have occurred when the gas enters the bar regions and the shock differences could be due to differences in the moving cloud masses. Based on tracers of ionizing photons, it is unlikely that the morphological differences are due to selective photo-dissociation of the molecules. We also observe direct heating of molecular gas in strongly shocked zones, with high $\mathrm{SiO} / \mathrm{HNCO}$ ratios, where temperatures are determined from the transitions of ammonia. Strong shocks appear to be the most efficient heating source of molecular gas, apart from high energy emission emitted by the central supermassive black hole Sgr A* and the processes within the extreme star formation region Sgr B2.
\end{abstract}

Keywords. ISM: molecules — Galaxy: center — radio lines: ISM

\section{Introduction}

The central molecular zone (CMZ) covers the inner $\sim 500 \mathrm{pc}$ of the Milky Way and contains a substantial amount of molecular gas (several $10^{7} \mathrm{M}_{\odot}$, e.g. Oka et al. 1998). Models show that the gas may be funneled along the central Galactic bar into the CMZ and eventually accretes on $x_{2}$ orbits that cover the inner $\sim 200 \mathrm{pc}$ (e.g. Morris \& Serabyn 1996). Eventually the gas will be destroyed by environmental influences, during the process of star formation, or, in rare cases, the dense gas will be transported all the way further toward Sgr A*, the central supermassive black hole of the Milky Way.

In this paper, we focus on material that is influenced by shocks. HNCO, for example, is a molecule that is formed on dust grains and liberated into the gas phase by weak shocks (Meier \& Turner 2005). Stronger shocks can be traced by $\mathrm{SiO}$ where the silicon is provided by shock destruction of the dust grains themselves (Martin-Pintado et al. 1997). Since the ISM conditions in the Galactic center are dominated by shock physics, we use these two molecules to trace the environment as the gas accumulates in the CMZ. Jones et al. (2012) present maps of both molecules using the ATNF/CASS Mopra† telescope and Ott et al. (2008) show single dish Mopra maps of ammonia, a molecule which is used as a thermometer to trace shock heating.

$\dagger$ The Mopra telescope is part of the Australia Telescope National Facility which is funded by the Commonwealth of Australia for operation as a National Facility managed by CSIRO. 


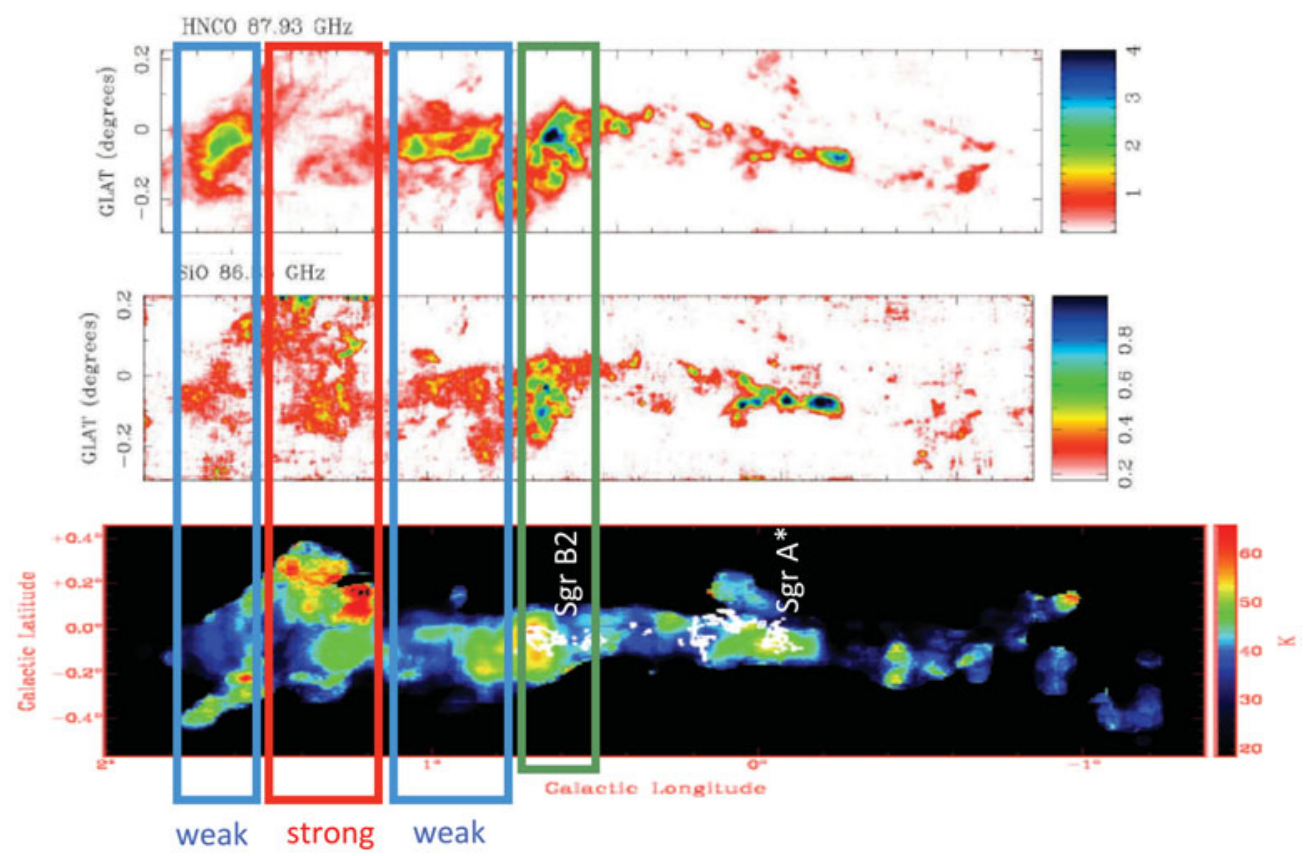

Figure 1. Shock tracers in the CMZ: top: $\mathrm{HNCO}$, middle: SiO. At the bottom we show a gas temperature map as derived from $\mathrm{NH}_{3}$. The regions with weak shocks are marked in blue, the strongly shocked regions in red. The green box centers on the star forming region Sgr B2 and marks the likely point where the $x_{1}$ orbits accrete on the $100 \mathrm{pc}$ ring, the location of the $x_{2}$ orbits. [A COLOR VERSION IS AVAILABLE ONLINE.]

\section{The shock structure of inflowing gas}

In Figure 1 we show the distribution of the $\mathrm{HNCO}\left(4_{04}-3_{03}\right)$ and $\mathrm{SiO} J=(2-1)$ line emission in the CMZ. In particular the regions east of Sgr B2 show a distinct pattern of alternating strong $\mathrm{SiO}$ and $\mathrm{HNCO}$ lines. This hints to alternating stronger and weaker shocks, where the shocks might have occurred at the time the gas entered the large Galactic bar or along $x_{1}$ orbits as the gas moves toward the Galactic center. Eventually the gas may accrete on the $100 \mathrm{pc}$ ring (likely the location of the $x_{2}$ orbits) that was discovered in Herschel dust maps (Molinari et al. 2011). This scenario is in agreement with gas heating by shocks. At the positions where the $\mathrm{SiO}$ emission dominates, the ammonia temperature map shows much higher temperatures, compared to the places with HNCO dominating. In addition, the velocity dispersion increases at the same morphological features. The shock heated gas is some of the hottest gas in the Galactic center, outside of the environments of compact sources.

\section{References}

Jones, P. A., Burton, M. G., Cunningham, M. R., et al. 2012, MNRAS 419, 2961

Martin-Pintado, J., de Vicente, P., Fuente, A., \& Planesas, P. 1997, ApJ Lett. 482, L45

Meier, D. S. \& Turner, J. L. 2005, ApJ 618, 259

Molinari, S., Bally, J., Noriega-Crespo, A., et al. 2011, ApJ Lett. 735, L33

Morris, M. \& Serabyn, E. 1996, ARAA 34, 645

Oka, T., Hasegawa, T., Sato, F., Tsuboi, M., \& Miyazaki, A. 1998, ApJS 118, 455

Ott, J., Henkel, C., Burton, W. A., et al. 2008, Gas and Stars in Galaxies - A Multi-Wavelength $3 D$ Perspective 